\title{
Molecular and Pathotypic Characterization of Fowl Adenovirus Associated with Inclusion Body Hepatitis in Indian Chickens
}

\section{Dasharath B. Shinde}

Symbiosis School of Biological Sciences, Symbiosis International (Deemed University), Gram: Lavale, Taluka: Mulshi, Pune-412115 https://orcid.org/0000-0003-4850-231X

Anil L Thormoth

Symbiosis School of Biological Sciences, Symbiosis International (Deemed University), Gram: Lavale, Taluka: Mulshi, Pune-412115 https://orcid.org/0000-0001-6697-0438

\section{Santosh S. Koratkar ( $\nabla$ santosh.koratkar@gmail.com )}

Symbiosis School of Biological Sciences, Symbiosis International (Deemed University), Gram: Lavale, Taluka: Mulshi, Pune-412115 https://orcid.org/0000-0002-9413-950X

\section{Neeti Sharma}

Symbiosis School of Biological Sciences, Symbiosis International (Deemed University), Gram: Lavale, Taluka: Mulshi, Pune-412115

\section{Ashok Rajguru}

JAPFA Comfeed India Pvt. Ltd. Sakore Nagar, Viman Nagar, Pune-411014,

\section{Vinay Rale}

Symbiosis School of Biological Sciences, Symbiosis International (Deemed University), Gram: Lavale, Taluka: Mulshi, Pune-412115

\section{Prasad Wagh}

JAPFA Comfeed India Pvt. Ltd. Sakore Nagar, Viman Nagar, Pune-411014

\section{Teguh Y. Prajitno}

P.T. Japfa Comfeed Indonesia Tbk., MT Haryono Kav. 16, Jakarta 12810, Indonesia

\section{Satish S. Tongaonkar}

Symbiosis School of Biological Sciences, Symbiosis International (Deemed University), Gram: Lavale, Taluka: Mulshi, Pune-412115

\section{Research Article}

Keywords: Fowl Adenovirus (FAdV), Inclusion Body Hepatitis (IBH), Serotype-11, SPF chicks, Histopathology, PCR, Hexon gene

Posted Date: August 26th, 2020 
DOI: https://doi.org/10.21203/rs.3.rs-64286/v1

License: (a) (i) This work is licensed under a Creative Commons Attribution 4.0 International License. Read Full License 


\section{Abstract}

Inclusion body hepatitis (IBH) is a disease of chickens characterized by acute mortality with severe anemia caused by fowl adenoviruses (FAdV). IBH is one of the most important re-emerging diseases world-wide. In India earlier IBH/ Hydropericardium Syndrome (HPS) outbreaks were mainly due to FAdV) type 4; however, in recent outbreaks, other serotypes are involved, despite regular vaccination in breeders and young chicks. The aim of the present study was isolation and molecular characterization of FAdV associated with IBH in India. A total of 193 liver samples of IBH suspected broiler chickens were collected from different regions of India. Liver samples were initially screened by histopathology and further, a total of 127 samples which microscopically showed basophilic, intra-nuclear inclusion bodies were processed for FAdV detection. A total of 69 samples were found to be positive for Hexon gene in PCR, of which 28 were sequenced. These samples showed more than $94 \%$ sequence homology with FAdV 2 and $97 \%$ with FAdV11, which was confirmed by Restriction Enzyme Analysis (REA). Tissue Culture Infective Dose 50 $\left(\mathrm{TCID}_{50}\right)$ and Egg Infective Dose ${ }_{50}$ (EID50) titer of isolated FAdV was found to be $10^{6.5} / \mathrm{ml}$. Pathogenicity study in SPF chicks showed $100 \%$ mortality up to Post Infection Day (PID)-6. These findings conclude that the prevalence of FAdV-11 is currently causing IBH outbreaks India. However, further genetic and pathogenic analysis of FAdV is required, which would provide useful information for the development of the efficient commercial IBH vaccine.

\section{Introduction}

Inclusion Body Hepatitis (IBH) is a disease of chicken characterized by acute mortality, often with severe anemia, caused by an adenovirus. In northern parts of India, IBH was reported in broilers with causing mortality $10-60 \%$ (Sreenivas Gowda and Satyanarayana 1994). Fowl adenoviruses (FAdVs) are main causative agents of IBH worldwide. Adenoviruses are non-enveloped icosahedral in shape, size in between 70 to $100 \mathrm{~nm}$ in diameter and containing a linear dsDNA of approximately $45 \mathrm{~kb}$ in size of its genome (Horne, et al. 1959). The major structural proteins are hexon and fiber proteins, which are noncovalently linked to the penton base, a structure known as penton (Parthiban et al. 2014). Isolation of FAdV is an important procedure for subsequent identification and classification of FADVs and its serotypes. Isolation can be done using primary chick embryo liver cell culture (CEL) or primary chicken embryo kidney (CEK) cells and SPF eggs (Singh et al. 2004). In India many outbreaks of IBH being reported, despite regular vaccination in breeders and young chicks.

The present study was designed to isolate and characterize the FAdVs in the current IBH outbreaks in Indian broiler chickens by PCR as well as using various in-vitro-and in-vivo studies.

\section{Materials And Methods}


Materials and reagents: The materials and reagents used in the present study were procured from HiMedia: Dulbecco's Modified Eagle's Medium (DMEM), Trypsin Phosphate Verses Glucose (TPVG), Trypsin-EDTA, Antibiotic solution (Penicillin, Streptomycin and Gentamicin), Fetal Bovine Serum (FBS), M199 Medium, Hanks Balanced Salt Solution (HBSS), Phosphate Buffer Saline (PBS) and Viral Transport Medium (VTM). Agilent: Taq DNA polymerase and Taq Buffer. Bangalore Genei: Asp1 restriction enzyme, dNTPs, 10X Buffer, 100bp DNA ladder, 6 X loading dye. QIAGEN: DNA Extraction Kit. Tarsons: Tissue culture flasks and tissue culture plates.

Sample Collection: Total of 193 liver tissue samples of IBH suspected broiler chickens were collected from different parts of India (Maharashtra, Telangana, Karnataka, and West Bengal). Liver samples from same flocks were pooled in VTM and transferred to the laboratory and were stored at $-80^{\circ} \mathrm{C}$ for further use.

Initial Screening: Initial screening of IBH was carried out based on clinical and necropsy findings and cytological examination by performing histopathology of collected liver samples.

Sample Processing. Approximately 1-2 grams of IBH positive 127 liver samples detected by histopathology were taken into a sterile mortar-pestle and gradually ground aseptically. $10 \%$ liver suspension was prepared in M-199 medium containing antibiotics (Gentamicin-10 $\mu \mathrm{g} / \mathrm{ml}$, Penicillin $100 \mathrm{U} / \mathrm{ml}$ and Streptomycin $100 \mu \mathrm{g} / \mathrm{ml}$ ) and three freeze-thaw cycles were given to the samples and were stored at $-80^{\circ} \mathrm{C}$ for further experiment.

Isolation of FAdV by Egg inoculation method: This experiment was carried out in 5-7 day old embryonated SPF chicken eggs (Cowen 1988). In brief, 10\% liver suspension was diluted 1:100 and 1:1000 in sterile PBS (pH-7.4) and $0.2 \mathrm{ml} / \mathrm{egg}$ was inoculated by yolk sac route. Eggs were further incubated at $37^{\circ} \mathrm{C}$, and daily mortality was observed and recorded.

Preparation of chick embryo liver (CEL) culture: CEL culture was prepared using 12-14 days-old embryonated SPF chicken eggs. Approximately $3 \times 10^{5} \mathrm{cells} / \mathrm{ml}$ were seeded in tissue culture flasks and incubated at $37^{\circ} \mathrm{C}$ in a $\mathrm{CO}_{2}$ incubator until a confluent monolayer was formed. (Soumyalekshmi, Ajith, and Meshram 2014)

Isolation of FAdV in CEL culture: Liver suspension, which was pre-confirmed for the presence of the Hexon gene by PCR, was inoculated on the monolayer CEL culture. Cells were daily observed for CPE till 3-4 days of post-infection. Subsequently, five passages were done in CEL culture, and finally, tissue culture fluid (TCF) were harvested using three freeze-thaw cycles and stored at $-80^{\circ} \mathrm{C}$ for further use.

Egg infectivity dose $\left(E I D_{50}\right)$ : EID $_{50}$ was determined by using different dilutions of liver suspension ranging from $10^{-3}$ to $10^{-8}$ in sterile PBS, and $0.2 \mathrm{ml}$ of each dilution were inoculated in SPF eggs. Control eggs were inoculated with $0.2 \mathrm{ml}$ PBS and incubated for 5-6 days post-inoculation at $37^{\circ} \mathrm{C}$. After incubation eggs were kept for chilling at $4^{\circ} \mathrm{C}$ for overnight and harvested aseptically. The liver was collected from all 
embryos, and presence of lesions was noted. EID ${ }_{50}$ was calculated by and Muench formula (Read and Muench 1938).

Tissue culture infectivity dose $\left(T C I D_{50}\right)$ : Different concentrations of liver suspension ranging from $10^{-3}$ to $10^{-7}$ were prepared in sterile PBS, and $100 \mu$ l of each dilution was inoculated on confluent monolayer of CEL cells for $1 \mathrm{~h}$ at $37^{\circ} \mathrm{C}$ in a $\mathrm{CO}_{2}$ incubator. After incubation $0.5 \mathrm{ml}$ of DMEM supplemented with $2 \% \mathrm{FBS}$ was added in each well, and plate was incubated again. The cells were observed daily for CPE, and $\mathrm{TCID}_{50}$ titer was calculated by Read and Muench formula (Read and Muench 1938).

Histopathology:For histopathological examination, liver and kidney from the IBH suspected chicks were collected in $10 \%$ buffered formalin, embedded in paraffin sectioned at 4um, stained with hematoxylin and eosin and examined by light microscopy (Okuda et al. 2001).

DNA extraction and Polymerase Chain Reaction (PCR): DNA was extracted from all 127 samples using the QIAGEN DNA extraction kit as per the manufacturer's instructions.

Sequencing: Amplified DNA products were gel-purified using Purelink PCR purification kit (Thermo Fisher Scientific) as per the manufacturer's instructions. The purified products were subjected to automated sequencing (Applied BiosystemsBigDye Terminator V3.1) retrived sequences of hexon gene then used tconstruct the phylogenetic tree (Barua et al. 2005).

Restriction Enzyme Analysis (REA): The PCR products generated was purified using Wizard ${ }^{\mathrm{TM}}$ PCR Preps DNA purification system (Promega, USA), and purified PCR product were subjected to digestion with restriction enzyme Aspl(Meulemans et al. 2001).

Pathogenicity study: The experiment was carried out in day-old SPF chicks as described previously (Junnu et al. 2015) with minor modifications. Briefly, the chicks were randomly allocated in six groups. The four groups $(n=8)$ were infected intrapeitonially with $0.2 \mathrm{ml}$ of $10^{-3}, 10^{-4}, 10^{-5}$, and $10^{-6}$ dilution of virus suspension containing $10^{-5.5} / \mathrm{ml}$ egg infectivity dose $\left(\operatorname{EID}_{50}\right)$. Fifth group $(n=8)$ birds kept as incontact with infected birds and in control group birds $(n=10)$ were administered with $0.2 \mathrm{ml}$ PBS and was kept in a separate isolator with same feed and housing conditions. Birds were clinically examined daily for mortality and any kind of sickness.

\section{Results And Discussion}

Hemboldt and Frazer in 1963, first time reported IBH disease in the USA. Till date, many countries recorded IBH in poultry (El-Tholoth and El-Azm 2019; McFerran and Adair 1977). IBH outbreaks in India, China, South Asia, South Korea, Japan, Mexico, North America, and Eastern Europe caused immense economic losses to the poultry industry worldwide (Niczyporuk 2017; Li et al. 2019). In recent years increasing mortality and poor growth rate are the main challenges in poultry industries in India, keeping this in mind, we examined liver samples of 193 IBH suspected commercial broiler chicks from different 
states of India such as Maharashtra, Karnataka, Telangana, and West-Bengal of which histopathologically positive samples were used for further study (Table.1). A similar study was reported from Canada in which 287 liver samples collected and characterized (Ojkic et al. 2008).

Liver suspension (10\%) were prepared in M-199 medium. DNA was extracted from each sample and further tested using PCR for the presence of the hexon gene (Hess 2010). The hexon protein consists of conserved pedestal regions and variable loops. In PCR, we found that $54.33 \%$ infected liver samples are positive for hexon gene (Table 1).

Table 1: IBH suspected chick liver sample collection details from different states of India.

\begin{tabular}{|llllll|}
\hline $\begin{array}{l}\text { Sr. } \\
\text { No }\end{array}$ & State & $\begin{array}{l}\text { Total sample } \\
\text { collected }\end{array}$ & $\begin{array}{l}\text { Positive in HP } \\
\text { screening }\end{array}$ & $\begin{array}{l}\text { PCR } \\
\text { positive }\end{array}$ & $\begin{array}{l}\text { Sequencing } \\
\text { done for }\end{array}$ \\
\hline 1 & $\begin{array}{l}\text { Maharashtra } \\
(\mathrm{MH})\end{array}$ & 132 & $98(74 \%)$ & $61(62 \%)$ & 23 \\
\hline 2 & $\begin{array}{l}\text { Karnataka } \\
(\mathrm{KN})\end{array}$ & 10 & $03(30 \%)$ & $03(100 \%)$ & 03 \\
\hline 3 & $\begin{array}{l}\text { Telangana } \\
(\mathrm{TL})\end{array}$ & 18 & $08(44 \%)$ & $05(62 \%)$ & 02 \\
\hline 4 & $\begin{array}{l}\text { West Bengal } \\
\text { (WB) }\end{array}$ & 33 & $18(54 \%)$ & $00(0 \%)$ & 00 \\
\hline & 193 & 127 & 69 & 28 \\
\hline
\end{tabular}

Amplified hexon gene DNA products were further sequenced. These were compared with sequences of FAdV-1 to FAdV-11 retrieved from GenBank (Table 2). The sequence similarity was identified by constructing phylogenetic analysis using ClustalW (Fig 1). Phylogenic analysis revealed that our sequence showed $>94 \%$ homology with FAdV-2 and $>97 \%$ homology with FAdV- 11 . Sequence obtained in the study have been submitted to GenBank under the accession number MK933773. Similar studies from different countries have reported sequence homology using phylogenetic analysis of the hexon gene sequence of FAdV for e.g., a study conducted in Egypt showed a close genetic relationship with UK (FAdV2,3) and Canada (FAdV-4, 5) (El-Tholoth \& El-Azm 2019).

Table 2: FAdV hexon gene sequences taken from GenBank with their accession numbers 


\begin{tabular}{|lll|}
\hline FAdVserotype (European classification) & GenBank Accession number & Reference \\
\hline FAdV 1 CELO & AF339914 & NCBI \\
\hline FAdV 2, ATCC VR-827 & AF339915 & S Barua et al.,2005 \\
\hline FAdV 2 & AF508946.1 & NCBI \\
\hline FAdV 2 & AF508947.1 & NCBI \\
\hline FAdV 3, ATCC VR-828 & AF339916 & S Barua et al.,2005 \\
\hline FAdV 4, ATCC VR-829 & AF339917 & S Barua et al.,2005 \\
\hline FAdV 4b & AF154246.1 & NCBI \\
\hline FAdV 5, ATCC VR-830 & AF339919 & S Barua et al.,2005 \\
\hline FAdV 6, ATCC VR-831 & AF339921 & S Barua et al.,2005 \\
\hline FAdV 7, ATCC VR-832 & AF339922 & S Barua et al.,2005 \\
\hline FAdV 8, ATCC VR-833 & AF339918 & S Barua et al.,2005 \\
\hline FAdV 9, ATCC VR-834 & AF339923 & S Barua et al.,2005 \\
\hline FAdV 10, ATCC VR-835 & AF339924 & S Barua et al.,2005 \\
\hline FAdV 11, strain X11 & AF339920 & S Barua et al.,2005 \\
\hline FAdV 11 & HQ697595 & NCBI \\
\hline FAdV 11 & FJ360748 Brazil & NCBI \\
\hline FAdV 12, strain 380 & AF339925 & S Barua et al.,2005 \\
\hline FAdV 12 & HM748587 HR4 & NCBI \\
\hline
\end{tabular}

REA was performed further to distinguish between the serotypes FAdV2 and FAdV11 using Asp/ (also known as Tth111I) restriction enzyme, as our sequence was showing high homology with both FAdV 2 and FAdV11. Aspl, which cut the 900bp product of FAdV11 into 448/452bp fragments and confirmed the presence of FAdV2 (Meulmans et al. 2001). The digested PCR products of hexon gene with restriction enzyme Asp1 which did not show any cut in DNA, hence we confirmed the involvement of FAdV11 in the current Indian IBH outbreaks.

Followed by molecular characterization, isolation of the FAdV was carried out for further confirmation studies. The eggs which were inoculated with FAdV isolates via yolk sac route were harvested on PID-4. The eggs were harvested and hemorrhagic embryo with IBH specific lesions on the liver was considered positive. The IBH specific lesions were more prominent in subsequent passages than the initial. The isolated FAdV has a titer of $10^{6.5} / \mathrm{ml} \mathrm{EID}_{50}$ as calculated by Reed and Muench method. 
Isolation of FAdV was also carried out in CEL cell culture. FAdV infected cells showed cytopathic effect (CPE) rounding, clumping, degeneration, and detachment of cells from monolayer at $72 \mathrm{~h}$ post-infection however no cytopathic effects (CPE) were observed in the untreated control CEL cultures (Fig. 2a). Complete detachment of cells monolayer was observed at $96 \mathrm{~h}$ post-infection, subsequent passages indicated CPE within 48 to $72 \mathrm{~h}$ post-infection (Fig. 2b).

After passage number five, tissue culture fluids (TCF) was harvested using three freeze-thaw cycles. DNA was extracted from TCF, and PCR was performed. PCR product showed amplification of the hexon gene at $897 \mathrm{bp}$ size. The amplified products were sequenced, and homology was observed with FAdV-11 isotype reported sequences on GenBank database. Infectivity titer of isolated virus was also determined in CEL culture. Tenfold serial dilutions of the isolated virus were prepared in sterile PBS ranging from $10^{-3}$ to $10^{-8}$ and cells monolayer inoculated with this different dilution of virus and CPE was observed. Plates were observed daily till PID-4. The $\mathrm{TCID}_{50}$ titer was calculated by Reed and Muench formula, and it was found to be $10^{6.5} / \mathrm{ml}$.

Pathogenicity was studied up to a period of seven days. It was found that at PID-7, $100 \%$ mortality was observed at $10^{-3}, 10^{-4}$ and $10^{-5}$ dilution, whereas in $10^{-6}$ dilution $50 \%$ mortality and in-contact birds $25 \%$ mortality was noticed. These results are similar to several other studies (Nakamura et al. 1999; Sochonewille et al. 2008; Zhao et al. 2015). One of the earlier reports showed a maximum of $80 \%$ mortality in SPF chicks in 5 days to post-infection with FAdV isolate (Alvarado et al. 2007). In yet another reported mortality was 30 to $100 \%$ (Ruan et al. 2018). The most prominent lesions were observed in the liver. An enlarged dystrophic liver with pale yellow discoloration, crumbly texture, hemorrhages, and visible necrotic foci was observed in both the infected and in-contact chick livers. These liver samples were further confirmed for the presence of the hexon gene by PCR, which was found to be positive for the hexon gene whereas, in control chicks no amplification for the hexon gene was observed.

During the pathogenicity studies experiment, chicks were sacrificed on PID-3, PID-6, and PID-22) and liver samples were collected in $10 \%$ formalin for histopathological evaluation. Histopathological examination revealed the most prominent lesions in the liver with the presence of intra-nuclear basophilic or eosinophilic inclusion bodies. Inclusion bodies were noticed in many hepatocytes, and hepatocytes degeneration and vacuolation were also observed (Fig. 3b). However, in control chicks IBH specific microscopic changes were not observed (Fig. 3a). These observations are similar to an earlier study (McFerran 1977). Further, the involvement of FAdV11 was confirmed by PCR amplification of hexon gene from these infected chicks.

Our findings conclude that the prevalence of FAdV-11 is currently causing IBH outbreaks in India. However, further genetic and pathogenic analysis of FAdV is required, which would provide useful information for the development of the efficient commercial IBH vaccine.

\section{Declarations}


Compliance with Ethics of Experimentation: The present study was approved by the Institutional Animal Ethical Committee (IAEC approved number: SSBS/IAEC/02-2016).

Conflict of Interest:The authors declare that they have no competing interests.

Acknowledgment: We would like to extend sincere thanks to Japfa Comfeed India Pvt. Ltd. Pune, for sponsoring this work. We are grateful to Symbiosis International (Deemed University), for giving permission to conduct this research at SSBS. We would like to thank Dr. K.A. Schat for critically reviewing and valuable inputs in manuscript writing. We also extend our thanks to Mr. Sanketkumar Nehul and Mr. Nilesh Chakankar for technical help to complete laboratory work.

\section{References}

1. Alvarado I R, Villegas P, El-Attrache J, Jensen E, Rosales G, Perozo F and Purvis L B. 2007. Genetic characterization, pathogenicity, and protection studies with an avian adenovirus isolate associated with inclusion body hepatitis. Avian Diseases 51(1): 27-32.

2. Barua S, Mondal B, Sanyal A, Hemadri D, Bandyopadhyay S K and Rai A. 2005. Sequencing and comparative analysis of hexon gene of fowl adenovirus 4 of Indian origin. Indian Journal of Biotechnology 4: 367-372.

3. Cowen B S. 1988. Chicken embryo propagation of type I avian adenoviruses. Avian Diseases: 32(2): 347-352.

4. El-Tholoth, Mohamed and Kamel I Abou El-Azm. 2019. Molecular detection and characterization of fowl adenovirus associated with inclusion body hepatitis from broiler chickens in Egypt. Tropical Animal Health and Production 51(5): 1065-1071.

5. Hess M. 2010. Detection and differentiation of avian adenoviruses: a review. Avian Pathology 29(3): 195-206.

6. Horne R W, Brenner S, Waterson A P and Wildy P. 1959. The icosahedral form of anadenovirus. Journal of Molecular Biology 1: 84-86.

7. Junnu S, Lertwatcharasarakul P, Jala V, Phattanakulanan S, Monkong A, Kulprasertsri S, Thivalai C, Chakritbudsabong W, Chaichoun K and Songserm T. 2015. An inactivated vaccine for prevention and control of inclusion body hepatitis in broiler breeders. The Thai Journal of Veterinary Medicine 45(1): 55-62.

8. Li G, Yu G, Niu Y, Cai Y and Liu S. 2019. Airborne transmission of a serotype 4 fowl adenovirus in chickens. Viruses 11(3): 262.

9. Meulemans G, Boschmans M, Van den Berg T P and Decaesstecker M. 2001. Polymerase chain reaction combined with restriction enzyme analysis for detection and differentiation of fowl adenoviruses. Avian Pathology 30(6): 655-660.

10. Nakamura K, Mase M, Yamaguchi S, Shibahara T and Yuasa N. 1999. Pathologic study of specificpathogen-free chicks and hens inoculated with adenovirus isolated from hydropericardium 
syndrome. Avian Diseases 43: 414-423.

11. Niczyporuk J S. 2017. Molecular characterisation of fowl adenovirus type 7 isolated from poultry associated with inclusion body hepatitis in Poland. Archives of Virology 162(5): 1325-1333.

12. Ojkic D, Martin E, Swinton J, Vaillancourt J P, Boulianne M and Gomis S. 2008. Genotyping of Canadian isolates of fowl adenoviruses. Avian Pathology 37(1): 95-100.

13. Okuda Y, Ono M, Yazawa S, Shibata I and Sato S. 2001. Experimental infection of specific-pathogenfree chickens with serotype-1 fowl adenovirus isolated from a broiler chicken with gizzard erosions. Avian Diseases 45(1): 19-25.

14. Parthiban M, Shalini K S, Divya K C, Kumanan K and Aarthi K S. 2014. Rapid detection of fowl adenovirus field samples using loop-mediated isothermal amplification assay. Indian Journal of Animal Sciences 84(1): 22-25.

15. Reed $\mathrm{L} J$ and Muench $\mathrm{H}$. 1938. A simple method of estimating fifty per cent endpoints. American Journal of Epidemiology 27(3): 493-497.

16. Ruan S, Zhao J, He Z, Yang H and Zhang G. 2018. Analysis of pathogenicity and immune efficacy of fowl adenovirus serotype 4 isolates. Poultry Science 97(8): 2647-2653.

17. Singh A, Kumari A, Maiti N K and Oberoi M S. 2004. Characterization of fowl adenoviruses isolated from field outbreaks of hydropericardium syndrome in Punjab. Indian Journal of Animal Sciences 74(5): 457-461.

18. Soumyalekshmi S, Ajith M K and Meshram C. 2014. Isolation of fowl adenovirus in chicken embryo liver cell culture and its detection by hexon gene based PCR. Indian Journal of Science Research and Technology 2(3): 33-36.

19. Sreennivas Gowda R N and Satyanarayana M C. 1994. Hydropericardium syndrome in poultry. Indian Journal of Veterinary Pathology 18: 159-61.

20. Zhao J, Zhong Q I, Zhao Y, Hu Y X and Zhang G. 2015. Pathogenicity and complete genome characterization of fowl adenoviruses isolated from chickens associated with inclusion body hepatitis and hydropericardium syndrome in China. Plos One 10(7): e0133073.

\section{Figures}




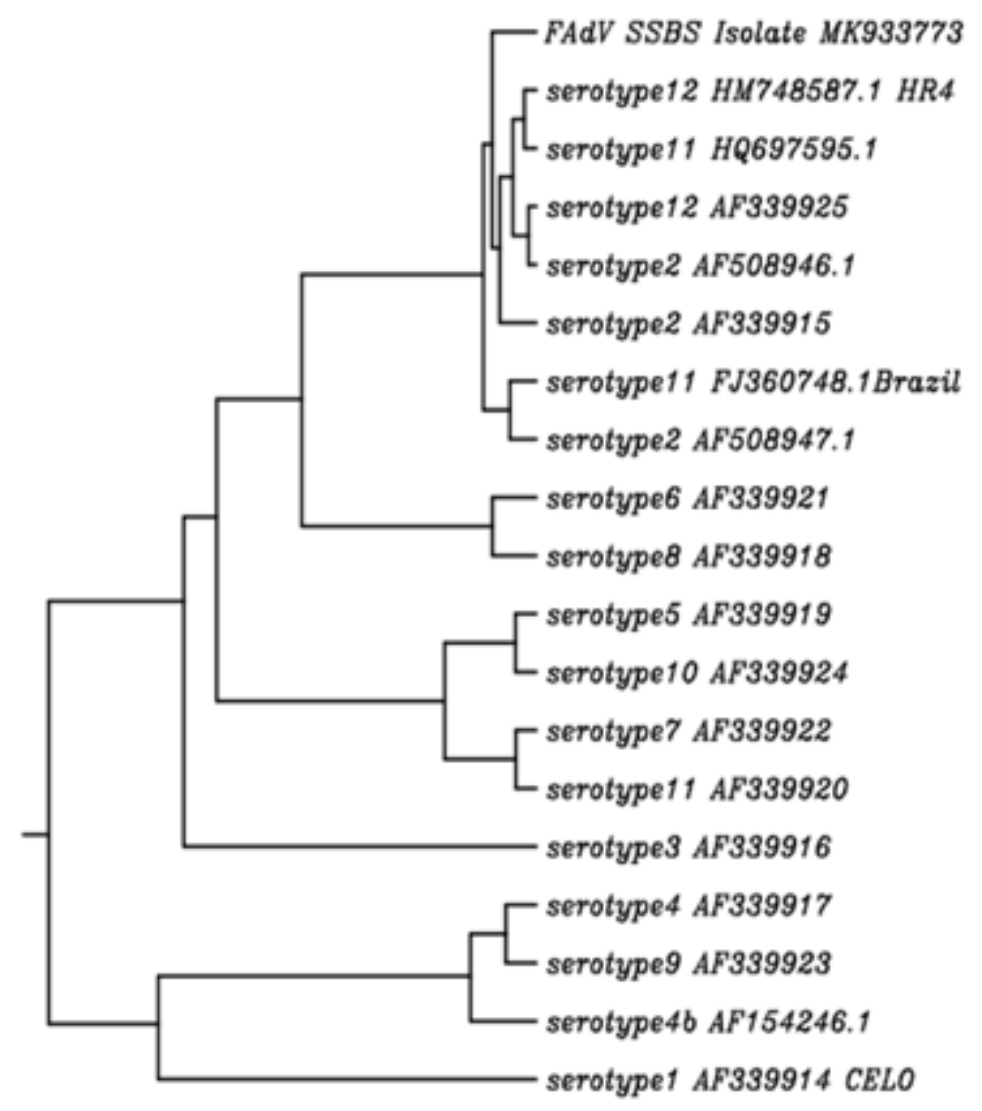

Figure 1

Phylogenetic analysis of Hexon gene sequence of FAdV SSBS isolate showing sequence homology with reported isolates at GenBank database.
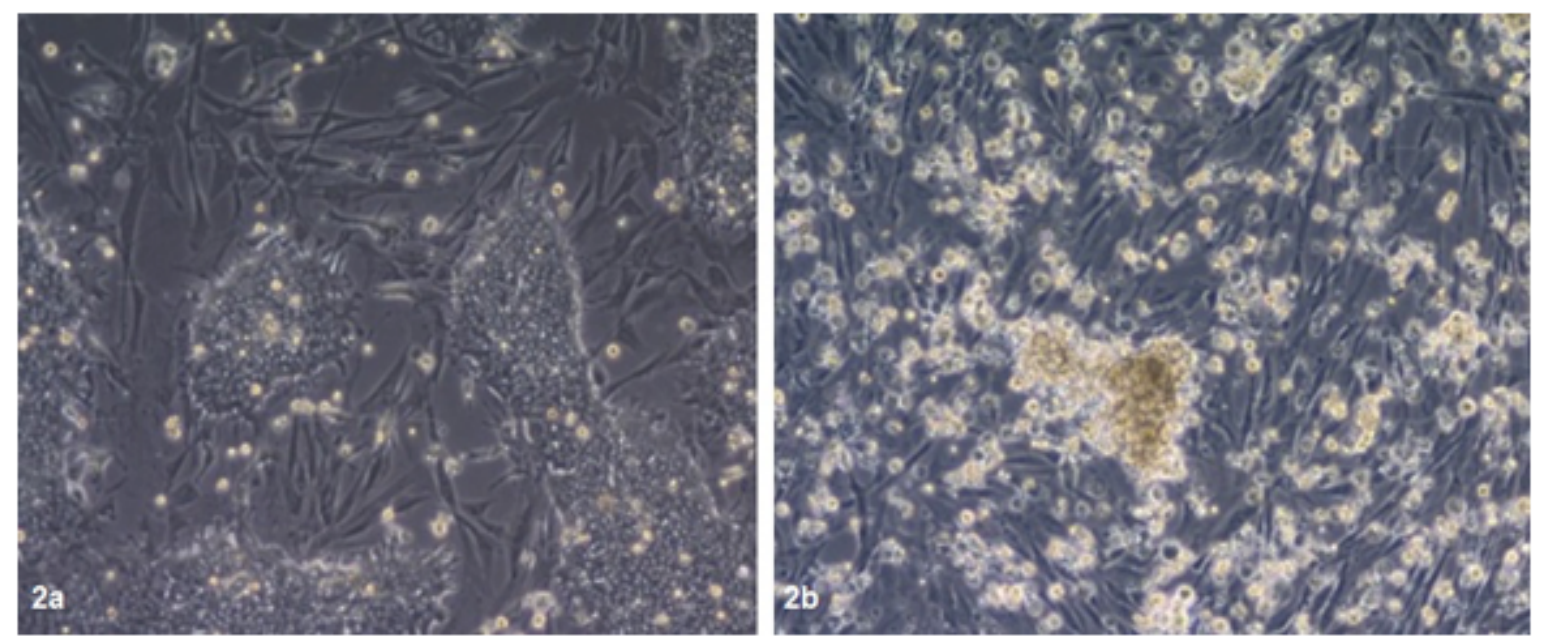

\section{Figure 2}

Cytopathic effect of FAdV on primary chick embryo liver cell culture. a: Primary chick embryo liver cell culture inoculated with PBS as control showing no cytopathic effect. b: Primary chick embryo liver cell culture inoculated with FAdV showing cytopathic effect at PID-3. 

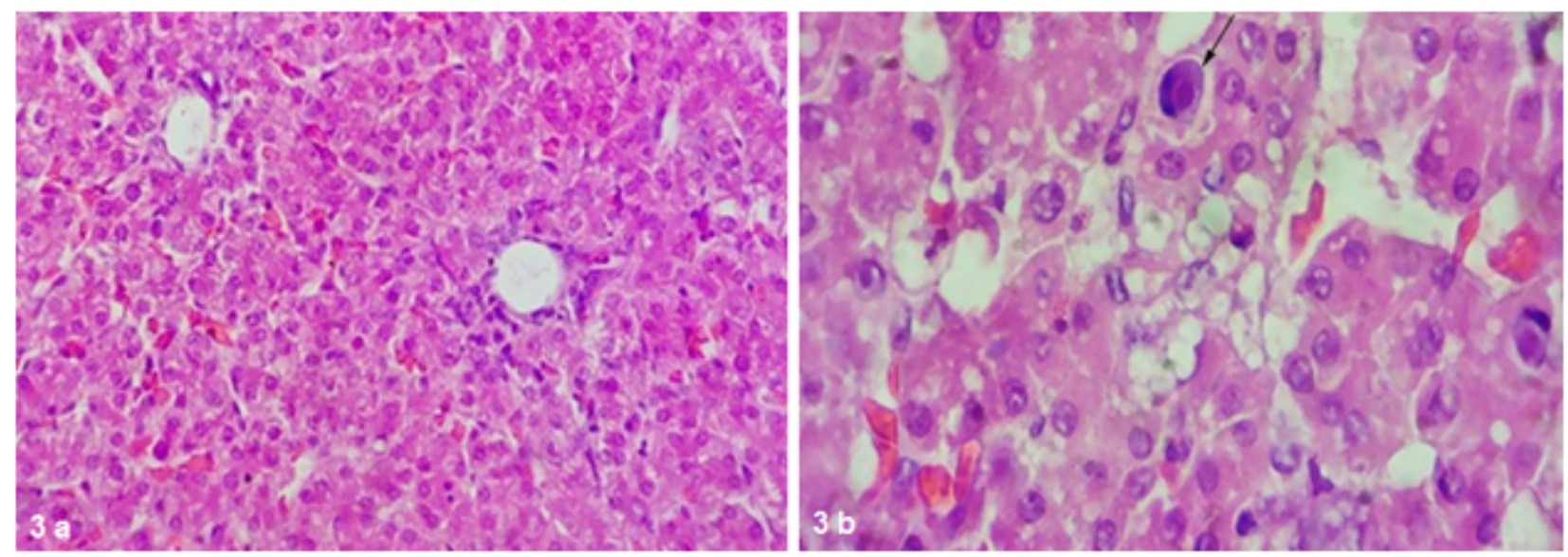

\section{Figure 3}

a: Control chick liver showing minimal focal hepatocytes degeneration (H\&E 40X); b: Virus-infected chick liver showing intra-nuclear basophilic inclusion bodies (Arrow) were noticed in many hepatocyte, hepatocytes degeneration and vacuolation also observed (H\&E 100X). 